ANDRIOLO, J.L.; WITTER, M; DAL ROSS, T.; GODÓI, R.S. Crescimento e desenvolvimento do tomateiro cultivado em substrato com reutilização da solução nutritiva drenada. Horticultura Brasileira, Brasília, v. 21, n. 3, p. 485-489, julho-setembro 2003.

\title{
Crescimento e desenvolvimento do tomateiro cultivado em substrato com reutilização da solução nutritiva drenada
}

\author{
Jerônimo L. Andriolo; Marcio Witter; Tiago Dal Ross; Rodrigo dos S. Godói \\ UFSM-CCR, Depto. Fitotecnia, 97105-900 Santa Maria-RS; E-mail: andriolo@creta.ccr.ufsm.br
}

\begin{abstract}
RESUMO
Dois experimentos foram conduzidos em ambiente protegido no outono e na primavera de 2001, com semeaduras em 17 de fevereiro e 3 de julho. $O$ transplante foi feito aos $41\left(1^{0}\right.$ experimento) e 36 dias ( $2^{0}$ experimento) após a semeadura, em sacolas de polietileno com $5,5 \mathrm{dm}^{3}$ de substrato comercial, instaladas no interior de calhas, com 3,3 plantas $\mathrm{m}^{-2}$. Os tratamentos constituíram-se de três soluções nutritivas, contendo o tratamento $\mathrm{T} 1$, em mol L-1: 0,04 de $\mathrm{KNO}_{3}$; 0,027 de $\mathrm{Ca}\left(\mathrm{NO}_{3}\right)_{2} ; 0,012$ de $\mathrm{MgSO}_{4}$, com adição de $1,5 \mathrm{~g} \mathrm{~L}^{-1}$ de superfosfato simples e solução de micronutientes. Em T1, forneceuse uma vez por semana o volume de $1 \mathrm{~L}$ de solução nutritiva por planta, totalizando $14,9 \mathrm{~g} \mathrm{~L}^{-1}$ de macronutrientes. Os tratamentos $\mathrm{T} 2$ e T3 consistiram na dose duplicada e triplicada T1, totalizando, respectivamente, 29,8 e 44,7 $\mathrm{g} \mathrm{L}^{-1}$ de macronutrientes, fornecidos também uma vez por semana. Os volumes drenados em cada irrigação foram recolhidos e reutilizados nas fertirrigações seguintes, completando-se as quantidades de fertilizantes necessárias para atingir as doses de cada tratamento. Os valores médios de condutividade elétrica (CE) da solução drenada foram de 3,$7 ; 6,8$ e $8,9 \mathrm{dS} \mathrm{m}^{-1}$ no primeiro e de 3,$3 ; 5,2$ e 7,4 $\mathrm{dS} \mathrm{m}^{-1}$ no segundo experimento, respectivamente em T1, T2 e T3. Entre os 40 e 82 dias após o transplante (DAT) no outono e os 37 e 79 DAT na primavera, foram feitas coletas de plantas para determinar o crescimento e o desenvolvimento. Na primavera, os frutos maduros nas plantas remanescentes foram colhidos e pesados para determinação da produtividade. Não foram observadas diferenças significativas no número de frutos entre os tratamentos. No outono, a massa seca total e vegetativa foi mais baixa no tratamento T3, enquanto a massa seca de frutos foi mais elevada nesse tratamento. Na primavera, as médias das duas primeiras variáveis foram mais baixas em $\mathrm{T} 3$, porém a massa seca de frutos não diferiu entre os tratamentos. A produtividade de frutos maduros decresceu com o aumento da CE. Concluiu-se que é possível reutilizar integralmente a solução nutritiva drenada no cultivo do tomateiro em substrato e que os efeitos negativos da CE elevada sobre a produtividade de frutos são observados somente com valores superiores a $4,9 \mathrm{dS} \mathrm{m}^{-1}$.
\end{abstract}

Palavras-chave: Lycopersicon esculentum, fertirrigação, cultivo sem solo, solução nutritiva.

\begin{abstract}
Growth and development of tomato plants in substrate with re-use of drained nutrient solution
\end{abstract}

Two experiments were carried out in a plastic greenhouse in autumn and spring 2001 . Sowing dates were February $17^{\text {th }}$ and July $3^{\text {rd }}$, respectively. At 41 ( $1^{\text {st }}$ experiment $)$ and 36 days ( $2^{\text {nd }}$ experiment $)$ after sowing, plantlets were transplanted to $5.5 \mathrm{dm}^{3}$ bags placed inside gullies, using a commercial substrate, in a plant density of 3.3 plants $\mathrm{m}^{-2}$. Treatments consisted of three nutrient solutions. In T1 treatment, fertilizer concentrations were, in mol. $\mathrm{L}^{-1}: \mathrm{KNO}_{3}, 0.04: \mathrm{Ca}\left(\mathrm{NO}_{3}\right)_{2}$, $0.027 ; \mathrm{MgSO}_{4}, 0.012$. Phosphorus (P) was added by $1.5 \mathrm{~g} \mathrm{~L}^{-1}$ of superphosphate $\left(20 \% \mathrm{P}_{2} \mathrm{O}_{5}\right)$, and micronutrients by a commercial mixture. For T1 plants, $1 \mathrm{~L}$ of the above nutrient solution was supplied once a week, containing $14.9 \mathrm{~g} \mathrm{~L}^{-1}$ of macronutrients. For T2 and T3 treatments the amounts of nutrients from $\mathrm{T} 1$ were applied in duplicate and triplicate, resulting in a total of macronutrient of 29.8 and $44.7 \mathrm{~g}$ $\mathrm{L}^{-1}$, respectively, supplied once a week. Drained volumes from each irrigation were collected and re-used in the next fertigations, after correcting nutrient concentrations in order to reach the original threshold level previously fixed for each treatment. Average electrical conductivity (EC) values of drained nutrient solution were $3.7 ; 6.8$ and $8,9 \mathrm{dS} \mathrm{m}^{-1}$ at the first and 3.3; 5.2 and $7.4 \mathrm{dS} \mathrm{m}^{-1}$ at the second experiment, respectively for T1, T2 and T3. From 40 to 82 days after planting date (DAP) in autumn and 37 to 79 DAP in spring. Plants were periodically harvested to determine growth and development. In spring, ripe fruits on remaining plants were harvested and weighed to determine fruit yield. No significant differences were found for the number of fruits among treatments. In autumn, total and vegetative dry mass were lower on $\mathrm{T} 3$ plants, whereas fruit dry mass was higher. In spring, means of total and vegetative dry mass were lower on $\mathrm{T} 3$ plants, but fruit dry mass did not differs among treatments. Fruit yield decreased by effect of increasing values of EC. Based upon these results we concluded that it is possible to reuse drained nutrient solutions, when growing the tomato crop in substrates, and negative effects of salinity on fruit yield are observed only for EC values up to $4.9 \mathrm{dS} \mathrm{m}^{-1}$.

Keywords: Lycopersicon esculentum, fertigation, soilless culture, nutrient solution.

(Recebido para publicação em 25 de abril de 2002 e aceito em 02 de junho de 2003)

$\mathrm{U}$ ma das principais limitações ao cultivo de plantas fora do solo diz respeito ao destino das soluções nutritivas descartadas. Na maior parte dos casos essas soluções são eliminadas no ambiente, em quantidades que podem atingir $2.300 \mathrm{~m}^{3} \mathrm{ha}^{-1}$ de água e $4.000 \mathrm{~kg}$ $\mathrm{ha}^{-1}$ de nutrientes, em culturas de hortaliças de ciclo longo como o tomateiro, o pepino e o meloeiro (Cortés, 1999). Faz-se necessário reduzir ao máximo essas quantidades, principalmente por razões de preservação ambiental, dentro do contexto atual em que se busca a sustentabilidade da produção agrícola a médio e longo prazo.

No cultivo de hortaliças em substratos, a composição da solução nutritiva a ser empregada é determinada levando-se em conta a concentração de absorção dos nutrientes pela planta e as interações entre os íons que compõem a solução nutritiva (Cañadas, 1999; Lopez, 1998; Lopez \& Alonso, 1998; Alpi \& Tognoni, 1999). Disso resulta que uma parte dos nutrientes fornecidos não é absorvida pela planta, a qual é 
acrescentada a outros elementos químicos de pequena ou nula absorção que estão presentes na água de irrigação, provocando o aumento da condutividade elétrica (CE) em torno das raízes (Sonneveld \& Welles, 1988). Um segundo fator que contribui para o aumento da concentração salina no interior do substrato é o fluxo de água da planta. Esse fluxo responde principalmente aos elementos do ambiente e ocorre de forma independente da absorção mineral. Quanto mais intenso o fluxo de água, mais alta a concentração salina da solução nutritiva contida no interior do substrato. Esses dois fenômenos servem de base para a prática da drenagem no cultivo de hortaliças em substrato, que deve ser ajustada de acordo com as condições ambientais a fim de manter a CE dentro dos limites adequados para cada cultura (Perez \& Lopez, 1998).

A reutilização da água e/ou solução nutritiva drenada nos cultivos em substrato pode implicar um aumento na $\mathrm{CE}$ da solução nutritiva fornecida. Entretanto, os valores de CE que podem ser atingidos em resposta às flutuações das variáveis do ambiente são difíceis de ser previstos. Níveis de salinidade no ambiente radicular superiores a 4,0 dS $\mathrm{m}^{-1}$ podem provocar redução do crescimento das plantas (Stanguellini et al., 1998; Li \& Stanguellini, 2001; RomeroAranda et al., 2001), queda no rendimento de frutos (Van Ieperen, 1996; Bolarin et al., 2001) e distúrbios fisiológicos como a podridão apical dos frutos (Cuartero \& Férnandez-Muñoz, 2001).

Determinou-se o crescimento e desenvolvimento do tomateiro cultivado em substrato com valores elevados de CE da solução nutritiva e a inferência sobre a possibilidade de reutilização da solução drenada no decorrer do ciclo da cultura.

\section{MATERIAL E MÉTODOS}

Dois experimentos foram conduzidos na UFSM, Rio Grande do Sul (latitude $29^{\circ} 43^{\prime} \mathrm{S}$, longitude $53^{\circ} 42^{\prime} \mathrm{W}$ e altitude $95 \mathrm{~m}$ ), no interior de um túnel alto de polietileno de $180 \mathrm{~m}^{2}$. Foi empregado o híbrido plurilocular Monte Carlo, com semeaduras em 17 de fevereiro e 3 de julho de 2001, em bandejas de poliestireno com 128 células, empregando substrato comercial. Aos 41 e 36 dias após a semeadura, respectivamente no outono e na primavera, as mudas com cinco folhas definitivas foram transferidas para sacolas de polietileno contendo 5,5 $\mathrm{dm}^{3}$ de substrato comercial orgânico (Plantmax $\left.{ }^{\circledR}\right)$, com uma planta por sacola e densidade de 3,3 plantas $\mathrm{m}^{-2}$. As sacolas foram dispostas no interior do túnel em calhas com $2 \%$ de declividade, revestidas com polietileno de baixa densidade de 150 $\mathrm{mm}$ de espessura. $\mathrm{O}$ espaçamento empregado foi de $1 \mathrm{~m}$ entre calhas e 0,30 $\mathrm{m}$ entre sacolas, as quais foram perfuradas na base para permitir a drenagem dos volumes de água fornecidos pela irrigação e que excederam a capacidade máxima de retenção de água do substrato. $\mathrm{Na}$ extremidade inferior de cada calha foi instalado um reservatório de polietileno com capacidade de 20 $\mathrm{L}$, destinado a recolher diariamente os volumes de solução nutritiva drenada. $\mathrm{O}$ volume recolhido foi armazenado em um segundo reservatório com capacidade de 100 L e posteriormente reutilizado na preparação da solução nutritiva. Sobre cada sacola foi instalado um tubo gotejador para o fornecimento de água e nutrientes, ajustando-se um gotejador no centro de cada sacola. As plantas foram conduzidas verticalmente com uma haste, através de fitas plásticas, e as ramificações axilares eliminadas uma vez por semana. O túnel foi ventilado nos dias ensolarados, através do soerguimento das extremidades laterais do filme de polietileno em até 1,20 m de altura, entre as $9 \mathrm{~h}$ e $18 \mathrm{~h}$, aproximadamente.

Do plantio até o final dos experimentos, os nutrientes foram fornecidos às plantas uma vez por semana, através da fertirrigação. Os tratamentos foram constituídos por três soluções nutritivas. Foi empregada como referência a solução nutritiva recomendada por Andriolo \& Poerscke (1997), para o cultivo do tomateiro em substrato, com a composição, em mol L-1: 0,04 de $\mathrm{KNO}_{3} ; 0,027$ de $\mathrm{Ca}\left(\mathrm{NO}_{3}\right)_{2} ; 0,012$ de $\mathrm{MgSO}_{4}$. O fósforo foi fornecido através da dissolução de $1,5 \mathrm{~g} \mathrm{~L}^{-1}$ de superfosfato simples, eliminando-se as impurezas por decantação. $O$ ferro foi fornecido na forma quelatizada, na proporção de $0,13 \mathrm{ml} \mathrm{L}^{-1}$
( $5 \%$ de $\mathrm{Fe}$ ), e os demais micronutrientes através da solução proposta por Jeannequin (1987), citada por Andriolo (1999). Foi fornecido o volume de $1 \mathrm{~L}$ de solução nutritiva por planta, totalizando $\quad 14,9 \quad \mathrm{~g} \quad \mathrm{~L}^{-1} \quad \mathrm{de}$ macronutrientes, em doses semanais, até o final do experimento. $\mathrm{O}$ tratamento $\mathrm{T} 1$ foi constituído pela dose de referência e os tratamentos T2 e T3 foram obtidos a partir da dose de T1, multiplicada por dois e três, respectivamente, totalizando 29,8 e 44,7 $\mathrm{g} \mathrm{L}^{-1}$ de macronutrientes. Em cada fertirrigação, a solução nutritiva a ser fornecida às plantas de cada tratamento foi preparada empregando-se a solução drenada estocada no respectivo reservatório. A CE dessa solução foi medida e a quantidade de nutrientes contida no volume de $1 \mathrm{~L}$ foi estimada pelo coeficiente 0,85 (Jeannequin, 1987). A quantidade de sais a adicionar foi obtida por diferença entre a quantidade total prevista originalmente para T1, T2 e T3 e aquela contida no volume de solução drenada a ser reutilizada. Essa quantidade foi dividida proporcionalmente entre os diferentes fertilizantes componentes da solução, de forma a preservar o equilíbrio eletroquímico existente na solução de referência. A irrigação foi feita sempre que necessária para repor os volumes de água perdidos pela transpiração, sendo suspensa aos primeiros sinais de escorrimento no fundo das sacolas.

O delineamento experimental empregado foi de blocos casualizados, com quatro repetições e 20 plantas por parcela. Quatro plantas de cada tratamento foram coletadas aleatoriamente, uma em cada parcela, aos $40 ; 47 ; 54 ; 61 ; 68 ; 75$ e 82 dias após o transplante (DAT) no outono e aos $37 ; 44 ; 51 ; 58 ; 65 ; 72$ e 79 na primavera. Imediatamente após a coleta, os órgãos vegetativos (folhas, caule, pecíolos, inflorescências e pedúnculos) e os frutos foram separados. Para estimar a área foliar da cultura, foram extraídos 100 discos de superfície igual a $0,5 \mathrm{~cm}^{2}$ de área, de folhas representativas das diferentes partes do dossel vegetativo. O material vegetal foi submetido a secagem em estufa de circulação forçada, a temperatura de $60^{\circ} \mathrm{C}$, até peso constante, para determinar a massa seca. Uma relação foi ajustada entre a massa e a área dos discos, que 
Tabela 1. Médias do número de folhas e frutos, índice de área foliar (IAF), massa seca vegetativa, de frutos e total nos experimentos 1 e 2. Santa Maria, UFSM, 2001.

\begin{tabular}{|c|c|c|c|c|c|c|}
\hline \multirow{2}{*}{ Tratamento } & \multicolumn{2}{|c|}{ Número $\left(m^{2}\right)$} & \multirow{2}{*}{$\begin{array}{c}\text { IAF } \\
\left(\mathrm{m}^{2} \mathrm{~m}^{-2}\right)\end{array}$} & \multicolumn{3}{|c|}{ Massa seca $\left(\mathrm{g} \mathrm{m}^{-2}\right)$} \\
\hline & Folhas & Frutos & & Frutos & Veget. & Total \\
\hline \multicolumn{7}{|l|}{ Outono } \\
\hline T1 & $85 a^{*}$ & $43 a$ & 3,9 a & $83,88 a b$ & $274,59 a$ & 358,47 a \\
\hline $\mathrm{T} 2$ & $83 a$ & $31 a$ & 3,9 a & $62,30 \quad b$ & $283,07 a$ & $345,37 a b$ \\
\hline T3 & $79 \quad b$ & $35 a$ & $3,3 \mathrm{~b}$ & $101,90 \mathrm{a}$ & 209,71 b & $311,61 \quad b$ \\
\hline $\mathrm{CV}(\%)$ & 2,98 & 22,25 & 6,81 & 10,11 & 8,26 & 6,56 \\
\hline \multicolumn{7}{|l|}{ Primavera } \\
\hline $\mathrm{T} 1$ & $83 a$ & $46 a$ & $3,5 \mathrm{a}$ & 243,81 a & 353,90 a & $597,71 \mathrm{ab}$ \\
\hline $\mathrm{T} 2$ & $85 a$ & 58 a & $3,8 \mathrm{a}$ & $247,01 \mathrm{a}$ & $375,99 a$ & $623,00 \mathrm{a}$ \\
\hline T3 & $78 \mathrm{a}$ & $48 a$ & $2,8 \quad b$ & $259,95 \mathrm{a}$ & $308,19 \quad b$ & $568,14 \quad b$ \\
\hline$\overline{C V}(\%)$ & 7,03 & 13,13 & 11,46 & 7,31 & 4,98 & 1,21 \\
\hline
\end{tabular}

*/Valores seguidos pela mesma letra na coluna não diferem entre si pelo teste de Duncan a 5\% de probabilidade.

foi empregada para estimar a área foliar a partir da massa seca de folhas. No experimento de primavera, os frutos maduros foram colhidos e pesados, para determinação da produtividade da cultura. A colheita iniciou aos 76 DAT e foi concluída aos 117 DAT, quando o experimento foi encerrado. As médias foram calculadas e submetidas a análise da variância, sendo a significância das diferenças testada pelo teste de Duncan, em nível de probabilidade de $5 \%$.

\section{RESULTADOS E DISCUSSÃO}

Os valores médios da $\mathrm{CE}$ durante o período de cada um dos experimentos, obtidos a partir das medidas diárias feitas na solução drenada de cada tratamento foram 3,$7 ; 6,8$ e $8,9 \mathrm{dS} \mathrm{m}^{-1}$ no experimento 1 , e 3,$2 ; 5,2$ e $7,4 \mathrm{dS} \mathrm{m}^{-1}$ no experimento 2, para T1, T2 e T3, respectivamente. As variações entre os experimentos são atribuídas à maior freqüência das irrigações feitas na primavera, quando é mais elevada a demanda hídrica da cultura. Uma maior freqüência de irrigações resulta em maior perda de nutrientes por lixiviação na solução drenada entre fertirrigações consecutivas.

$\mathrm{O}$ número de folhas por unidade de área de solo no outono mostrou valores inferiores nas plantas de T3, com diferenças significativas em relação a $\mathrm{T} 2 \mathrm{e}$ T1. Os valores máximos atingidos ao final do experimento foram de 85; $83 \mathrm{e}$ 79 folhas $\mathrm{m}^{-2}$, respectivamente para $\mathrm{T} 1$,
T2 e T3 (Tabela 1). Na primavera, os valores foram similares entre os tratamentos, sem diferenças significativas, e os valores máximos foram de $83 ; 85 \mathrm{e}$ 78 folhas $\mathrm{m}^{-2}$, respectivamente para $\mathrm{T} 1$, T2 e T3, ao final do experimento. Quanto ao número de frutos, no outono as plantas de T2 evidenciaram valores inferiores aos outros dois tratamentos, embora sem diferenças significativas, totalizando ao final do experimento 43; 31 e 35 frutos $\mathrm{m}^{-2}$, respectivamente para T1, T2 e T3 (Tabela 1). Na primavera, as plantas de T2 apresentaram o valor mais elevado para essa variável ao final do experimento, de 58 frutos $\mathrm{m}^{-2}$, enquanto os valores em T1 e T2 foram de 46 e 48 frutos $\mathrm{m}^{-2}$, respectivamente, porém sem diferenças significativas (Tabela 1).

A evolução do índice de área foliar da cultura foi similar àquela dos órgãos vegetativos, com valores mais baixos em T3, em ambos os experimentos (Figura 1ab). Ao final de cada período experimental, os valores estimados foram de 3,$9 ; 3,9$ e $3,3 \mathrm{~m}^{2} \mathrm{~m}^{-2}$, no outono e de 3,5; 3,8 e $2,8 \mathrm{~m}^{2} \mathrm{~m}^{-2}$ na primavera, respectivamente para T1, T2 e T3 (Tabela 1).

A massa seca dos órgãos vegetativos apresentou valores similares nos três tratamentos até os 61 DAT no outono e os 51 DAT na primavera. Ao final do período experimental, os valores foram de 274,$59 ; 283,07$ e $209,71 \mathrm{~g} \mathrm{~m}^{-2}$, no outono e de 353,$90 ; 375,99$ e $308,19 \mathrm{~g} \mathrm{~m}^{-2}$, na primavera, respectivamente para $\mathrm{T} 1$,
T2 e T3, diferindo significativamente de T1 e T2 (Tabela 1 Figura 2ab). Quanto a massa seca de frutos, no outono as plantas de T2 evidenciaram valores inferiores, porém as diferenças não foram significativas em relação a T1 (Figura 2c e Tabela 1). Na primavera, os valores foram similares entre os tratamentos, sem diferenças significativas em cada uma das coletas efetuadas (Figura 2d). Os valores máximos dessa variável ao final da cada experimento foram 83,$88 ; 62,30$ e $101,90 \mathrm{~g} \mathrm{~m}^{-2}$ no outono e 243,$81 ; 247,01$ e $259,95 \mathrm{~g} \mathrm{~m}^{-2}$ na primavera (Tabela 1). A produtividade de frutos maduros na primavera foi de 10,2 ; 11,1 e $8,6 \mathrm{~kg} \mathrm{~m}^{-2}$, respectivamente para T1, T2 e T3. Esses dados se ajustaram a um modelo quadrático: $\mathrm{Y}=$ $-0,36 x^{2}+3,45 x+2,95, R^{2}=0,99$, com valor máximo obtido para uma condutividade elétrica de 4,9 dS m-1.

Tem sido demonstrado na literatura que a produtividade de frutos do tomateiro depende simultaneamente do número de frutos por planta e da fixação de assimilados para sustentar o crescimento desses frutos (Bertin, 1993, 1995; Heuvelink, 1996; Konning, 1994). Por sua vez, a fixação de assimilados depende do número de folhas até atingir o IAF ótimo necessário para maximizar a intercepção de radiação solar e a fotossíntese da cultura (Heuvelink, 1996, Challa \& Baker, 1998). Resultados disponíveis na literatura mostraram que tanto o número de folhas como de 


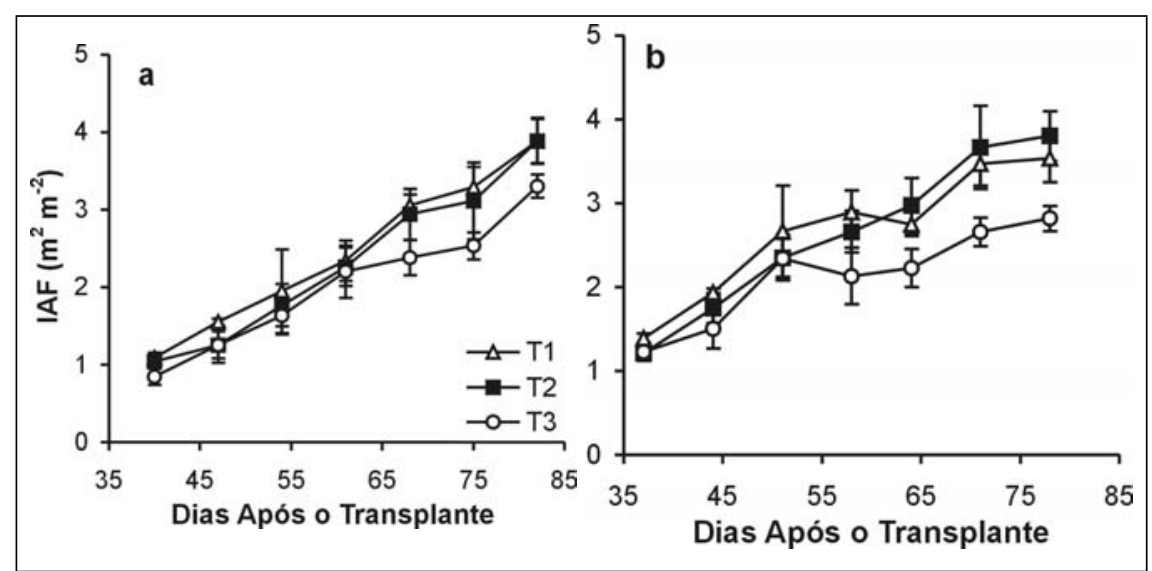

Figura 1. Índice de área foliar (IAF) de plantas de tomateiro cultivadas em substrato com reutilização da solução drenada em três níveis de condutividade elétrica, no outono (a) e na primavera (b). Santa Maria, UFSM, 2001.

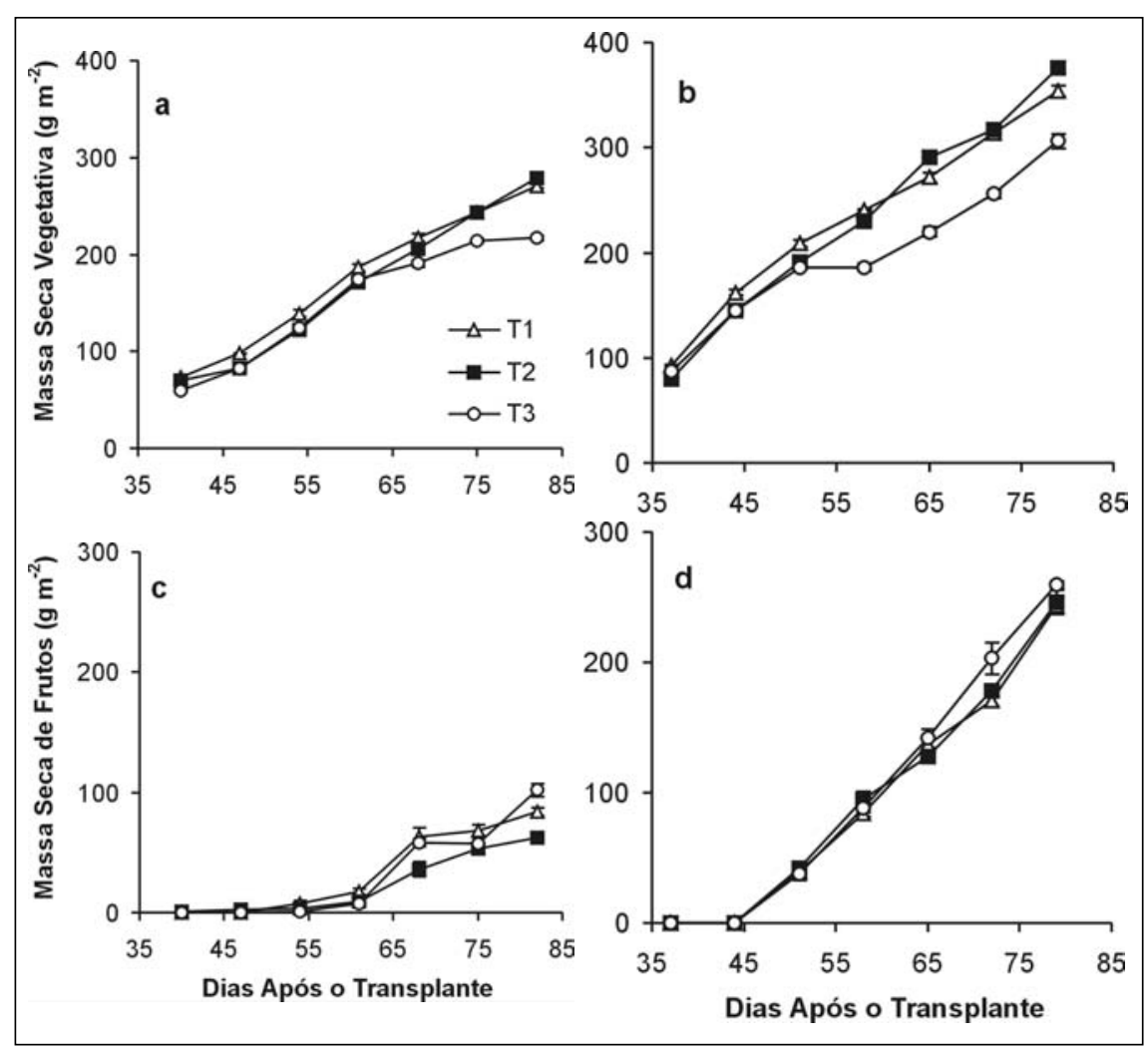

Figura 2. Massa seca vegetativa $(a, b)$ e de frutos $(c, d)$ de plantas de tomateiro cultivadas em substrato com reutilização da solução drenada em três níveis de condutividade elétrica, no outono (a,c) e na primavera (b,d). Santa Maria, UFSM, 2001.

frutos são reduzidos somente sob níveis de salinidade elevados, superiores a $9 \mathrm{dS}$ $\mathrm{m}^{-1}$ (Van Ieperen 1996; Li \& Stanghellini, 2001). Entretanto, esse efeito depende também do período de tempo durante o qual as plantas foram submetidas à salinidade. Nos resultados de Van Ieperen (1996) a redução ocorreu somente após 140 dias com CE de $9 \mathrm{dS} \mathrm{m}^{-1}$. Os dados obtidos no atual experimento confirmam essas conclusões. Indicam ainda, que nas culturas em ambiente protegido de ciclo curto como aquelas praticadas no Sul do Brasil no outono e na primavera, é pouco provável que a elevação da $\mathrm{CE}$ pela reutilização da solução drenada venha a interferir no número de folhas e de frutos da cultura.
Os resultados mostrados na literatura sugerem que o efeito mais pronunciado da salinidade do meio radicular ocorre sobre a expansão das folhas e o crescimento dos órgãos vegetativos, principalmente as folhas (van Ieperen, 1996; Stanguellini et al., 1998). Nos dois experimentos que foram realizados, esse efeito foi constatado somente nas plantas de T3, cujo valor médio da $\mathrm{CE}$ durante o período experimental foi de 8,86 $\mathrm{dS} \mathrm{m} \mathrm{m}^{-1}$ no outono e $7,44 \mathrm{dS} \mathrm{m}^{-1}$ na primavera. No experimento de primavera, o IAF máximo das plantas de T3 ao final do experimento foi de $2,9 \mathrm{~m}^{2} \mathrm{~m}^{-2}$, abaixo do limite ótimo, que é da ordem de $3,5 \mathrm{~m}^{2} \mathrm{~m}^{-2}$ (Konning, 1994). É importante salientar que aos 79 DAT, quando a coleta de plantas para a determinação da massa seca e da área foliar foi encerrada, a colheita de frutos já havia sido iniciada na primeira inflorescência. Desse período em diante a área foliar da cultura foi mantida mais ou menos constante pela retirada das folhas basais, como recomendado na produção comercial dessa cultura (Nuez, 1995). É surpreendente, por isso, que uma redução de $26 \%$ no IAF não tenha afetado a acumulação de massa seca nos frutos, a qual não diferiu entre os três tratamentos. Esse resultado confirma as conclusões anteriores de Andriolo \& Falcão (2000), os quais indicaram que uma parte da área foliar das plantas de tomateiro em ambiente protegido pode ser eliminada, sem diminuir a produção da massa seca de frutos da cultura.

Apesar de a massa seca de frutos não ter sido influenciada pela $\mathrm{CE}$, a produtividade de frutos foi reduzida em $22,7 \%$ quando a CE passou de $5,25 \mathrm{dS} \mathrm{m}^{-1} \mathrm{em}$ $\mathrm{T} 2$, para $7,44 \mathrm{dS} . \mathrm{m}^{-1}$, em T3. Essa falta de sincronia entre a acumulação de massa fresca e seca tem sido atribuída à diminuição na absorção e acumulação de água nos frutos, especialmente na fase de expansão celular (Cuartero \& Fernández-Muñoz, 1999; Bolarin et al., 2001). Esse efeito seria ainda mais pronunciado em materiais genéticos de frutos grandes, como aquele empregado nos atuais experimentos. Para minimizar esse efeito, o uso de materiais de frutos médios e/ou pequenos é recomendado. Essa prática permitiria associar produtividade e qualidade dos frutos, pois a 
salinidade aumenta a acidez e o teor de sólidos solúveis dos frutos, que são características qualitativas desejáveis em frutos de tomateiro (Cuartero \& Fernández-Muñoz, 1999).

Observando-se dados apresentados nesse trabalho, conclui-se que é possível reutilizar a solução nutritiva drenada no cultivo do tomateiro em substrato, segundo o procedimento adotado atualmente, que usa a fertirrigação a intervalos periódicos de tempo. No caso de essa prática implicar elevação da CE em torno das raízes, é pouco provável que efeitos negativos sobre a produtividade de frutos venham a ocorrer, desde que os valores não ultrapassem a 4,9 $\mathrm{dS} \mathrm{m}^{-1}$.

\section{AGRADECIMENTOS}

Os autores agradecem a FAPERGS e ao Programa PIBIC-CNPq, pela concessão de Bolsas de Iniciação Científica aos acadêmicos de Agronomia Marcio Witter e Tiago Dal Ross, respectivamente, no ano de 2001.

\section{LITERATURA CITADA}

ALPI, A.; TOGNONI, F. Cultivo en invernadero. Madrid: Mundi-Prensa, 3 ed. 1999. 347 p.

AMOR, F.M.; FLORES, P.; CARVAJAL, M.; MARTÍNEZ, J.M.; NAVARRO, J.M.; CERDÁ, A. Yield responses of soilless melon and tomato to different irrigation water qualities. Acta Horticulturae, n. 559, p. 333-338, 2001.

ANDRIOLO, J.L. Fisiologia das culturas protegidas. Santa Maria, Editora da UFSM, 1999. 142 p.
ANDRIOLO J. L.; FALCÃO, L. L. Efeito da poda de folhas sobre a acumulação de matéria seca e sua repartição para os frutos do tomateiro cultivado em ambiente protegido. Revista Brasileira de Agrometeorologia, Santa Maria, v. 8, p. 75-83, 2000. ANDRIOLO, J.L.; POERSCKE, P.R. Cultivo de tomateiro em substratos. Santa Maria: CCR, UFSM. 1997. 13 p. (Informe Técnico, 2).

BERTIN, N. Environnement climatique, competition pour les assimilats et modélisation de la nouaison de la tomate en culture sous serre. Paris: Institut National Agronomique ParisGrignon, 1993. 120p. (Thèse doctorat).

BERTIN, N. Competition for assimilates and fruit position affect fruit set in indeterminate greenhouse tomato. Annals of Botany, v. 75, p. 5565, 1995.

BOLARIN, M.C.; ESTAÑ, M.T.; CARO, M.; ROMERO-ARANDA, R.; CUARTERO, J.

Relationship between tomato fruit growth and fruit osmotic potential under salinity. Plant Science, v. 160, p. 1153-1159, 2001.

CAÑADAS, J.J.M. Sistemas de cultivo en substrato: a solución perdida y com recirculación del lixiviado. In: MILAGROS, M.F.; GÓMEZ, I.M.C. (ed.). Cultivos sin suelo II. Curso Superior de Especialización. Almería: DGIFA-FIAPACaja Rural de Almería. 1999, 2 ed. p. 173-205.

CHALLA, H.; BAKKER, J. Potential production within the greenhouse environment. In: ENOCH, Z.; STANHILL, G. eds. Ecosystems of the world. Amsterdan: Elsevier, 1998. p. 333-348.

CORTÉS, E.M. Características del riego en cultivos sin suelo: exigencias en aportación y manejo. Resultados experimentales en cultivo de pepino en perlita. In: FERNANDÉZ, M.F.; GÓMEZ, I.M.C., ed. Cultivos sin suelo II. Almeria: DGIFA/ FIAPA/Caja Rural de Almeria. 1999. p. 287-305. CUARTERO, J.; FERNANDÉZ-MUÑOZ, R. Tomato and salinity. Scientia Horticulturae, n. 78, p. 83-125, 1999.

HEUVELINK, E. Tomato growth and yield: quantitative analysis and synthesis. Wageningen: Wageningen Agricultural University, 1996.326 p. Dissertation.
JEANNEQUIN, B. Conduite de la fertilisation des cultures hors sol en maraîchage. PHM Revuede Horticultur, v. 275, p. 19-28. 1987.

KONNING, A.N.M. Development and dry matter distribution in glasshouse tomato: a quantitative approach. Wageningen: Wageningen Agricultural University, 1994. 240 p. Dissertation.

LI, Y.L.; STANGHELLINI, C. Analysis of the effect of EC and potential transpiration on vegetative growth of tomato. Scientia Horticulturae, n. 89, p. 9-21, 2001.

LOPEZ, C.C. Fertirrigación: aspectos básicos. In: LOPEZ, C.C. ed. Fertirrigación. Cultivos horticolas y ornamentales. Madrid: Mundi-Prensa. 1998, p. 65-79.

LOPEZ, C.C.; ALONSO, E.E. Calculo y preparación de disoluciones fertilizantes. In: LOPEZ, C.C. ed. Fertirrigación. Cultivos horticolas y ornamentales. Madrid: Mundi-Prensa. 1998, p. 125-172.

NUEZ, F. El cultivo del tomate. Madrid: MundiPrensa, 1995. 793 p.

ROMERO-ARANDA, R.; SORIA, T.; CUARTERO, J. Tomato plant-water uptake and plant-water relationships under sline growth conditions. Plant Science, n. 160, p. 265-272, 2001. PEREZ, M.L.S.; LOPEZ, C.C. Fertirrigación de cultivos hortícolas. In: LOPEZ, C.C. ed. Fertirrigación. Cultivos hortícolas $y$ ornamentales. Madrid: Mundi-Prensa. 1998, p. 374-389.

SONNEVELD, C.; WELLES, W.H. Yield and quality of rockwool-grown tomatoes as affected by variantions in EC-value and climatic conditions. Plant and Soil, n. 111, p. 37-42, 2001. STANGHELLINI, C.; VAN MEURS, W.T.M.; CORVER, F.; VAN DULLEMEN, E.; SIMONSE, L. Combined effect of climate and concentration of the nutrient solution on a greenhouse tomato crop. II: Yield quantity and quality. Acta Horticulturae, v. 458, p. 231-237, 1998.

VAN IEPEREN, W. Effects of different day and night salinity levels on vegetative growth, yield and quality of tomato. Journal of Horticultural Science, n. 71, p. 99-111, 1996. 